\title{
Montaje de un Banco de Pruebas para Medir Esfuerzos de Contacto y Vibración en Pares de Engranes
}

\author{
Assembly of a Test Bench to Measure Contact Stresses and Vibrations in \\ Gear Pairs \\ J. O. Dávalos-Ramírez¹, F. Sánchez Palma², A. Villanueva-Montellano¹, M. A. Lira-Martínez ${ }^{1}$ \\ ${ }^{1}$ Universidad Autónoma de Ciudad Juárez \\ ${ }^{2}$ Instituto Tecnológico de Pachuca
}

\section{RESUMEN}

En esta investigación se propone el montaje de un banco de pruebas para la medición experimental de esfuerzos y vibraciones en pares de engranes en contacto. La medición de los esfuerzos de contacto se realizó usando película de presión sensible al contacto, mientras que la de las vibraciones se hizo con un acelerómetro inalámbrico. Se midieron bajo diferentes velocidades de rotación: 1000, 1250 y 1500 rpm, en condiciones libre de carga y aplicando un torque de frenado de $27 \mathrm{Nm}$ en el eje de salida de los engranes. Los esfuerzos de contacto se incrementan bajo condiciones de carga y a altas revoluciones. En las mismas condiciones, la amplitud de las vibraciones disminuye, sin embargo, aumenta la frecuencia de los armónicos. Si bien estos resultados eran esperados, garantizan la efectividad del uso del banco de pruebas, el cual puede ser empleado para el diseño de nuevos engranes, el análisis de engranes que se encuentren en funcionamiento o el diagnóstico de fallas de una manera fácil y con una menor cantidad de componentes que los reportados en trabajos previos.

PALABRAS CLAVE: Engranes; esfuerzos de contacto; vibraciones; diseño mecánico.

\begin{abstract}
In this research we propose the assembly of a test bench to measure experimentally contact stresses and vibrations in gear pairs during meshing. The measurement of stresses was made using pressure film contact sensitive, whereas vibrations were measured trough a wireless accelerometer. Measurements were performed at 1000, 1250 and $1500 \mathrm{rpm}$ under free load conditions and with a brake torque of $27 \mathrm{Nm}$. Contact stresses increase when load is applied and running at high revolutions. Under the same conditions, vibrations amplitude decreases, but harmonics frequency increases. Although these results were expected, they confirm the reliability of the test bench which can be used to new gear designs, analysis of used gears or failure diagnosis in an easy way and with a smaller number of devices than those reported in previous works.
\end{abstract} KEYWORDS: Gears; contact stresses; vibrations; mechanical design.

Correspondencia:

DESTINATARIO: José Omar Dávalos Ramírez INSTITUCIÓN: Instituto de Ingeniería y Tecnología de la Universidad Autónoma de Ciudad Juárez DIRECCIÓN: Avenida del Charro núm. 450 norte, col. Partido Romero, C. P. 32310, Ciudad Juárez, Chihuahua, México. CORREOELECTRÓNICO: jose.davalos@uacj.mx
Fecha de recepción:

30 de julio de 2019

Fecha de aceptación:

24 de septiembre de 2019 


\section{INTRODUCCIÓN}

El diseño de engranes es bastante complejo y requiere, además de los cálculos analíticos de diseño, la realización de pruebas que garanticen su funcionalidad [1]. Dentro de los parámetros a analizar en un engrane se encuentran los esfuerzos de contacto y las vibraciones mecánicas. Los primeros se presentan al momento del acoplamiento de un par de engranes, provocan daños por picadura en los dientes y reducen la vida útil de los engranes.

En diversas investigaciones se han utilizado simulaciones numéricas aplicando el método del elemento finito para obtener la distribución de los esfuerzos de contacto en varios tipos de engranes ${ }^{[2,3]}$. El uso de este método simplifica el diseño de engranes, sin embargo, para garantizar la certeza de sus resultados, estos requieren de validación experimental.

Las principales técnicas experimentales encontradas en la literatura para la medición de esfuerzos de contacto emplean galgas extensiométricas y técnicas fotoelásticas. El uso de las primeras presenta dos desventajas principales: el resultado es puntual y de ellas se obtienen valores de deformación, $\varepsilon$, por lo que se requiere procesamiento posterior para convertir dicho resultado a esfuerzo a través de las ecuaciones correspondientes ${ }^{[4]}$, y respecto a las segundas, debido al montaje del arreglo experimental, visualizar la concentración de esfuerzos a través de líneas isocromáticas solo es posible en la parte frontal y no en la línea de contacto entre engranes, donde los esfuerzos de este tipo ocurren con magnitudes mayores, siendo esta zona crítica para su funcionamiento ${ }^{[5]}$.

Los diferentes tipos de fallas que pueden presentar un par de engranes durante su acoplamiento, tales como desbalanceo, desgaste en sus superficies, fractura en los dientes, etc., tendrán un comportamiento vibratorio característico que puede obtenerse a través del estudio de la señal de vibración de los engranes ${ }^{[6]}$. Las señales se adquieren a través de sensores conocidos como acelerómetros, que envían la señal medida a través de cables de tipo BNC conectados a un sensor de datos que se visualizan después en un monitor. Lo anterior representa una desventaja, ya que, al ser los engranes equipo rotatorio, el cableado necesita adecuaciones especiales en el banco de pruebas para la medición de señales, además del incremento en los componentes debido a los requerimientos para su manejo y adecuación.

En este trabajo se presenta el montaje de un banco de pruebas usando equipo de sensado especial con la finalidad de simplificar la medición de esfuerzos y vibraciones en pares de engranes durante su acoplamiento. Para la medición de esfuerzos de contacto se utilizó una película de presión sensible al contacto, la cual fue colocada en los dientes de los engranes, y para la de vibraciones un acelerómetro inalámbrico. Se aplicó un torque de frenado calculado mediante la colocación de galgas extensiométricas en el eje de salida del par de engranes. Además de las condiciones de carga, las mediciones se realizaron a diferentes velocidades de rotación.

\section{METODOLOGÍA}

El banco de pruebas propuesto cuenta con un motor de $1 \mathrm{hp}$ con una velocidad constante de $3600 \mathrm{rpm}$, al cual le fue conectado un variador de frecuencia con el fin de poder cambiar la velocidad de constante a variable. Se fijó un sistema de transmisión mediante una banda con poleas en el eje del motor y en el eje de entrada al tren de engranes. Los engranes cilíndricos de dientes rectos se encuentran en modo reductor de velocidad, piñón-rueda, con una relación $i=3$, montados en la segunda etapa de la transmisión, de tipo continua variable, de un vehículo todo terreno. Los ejes en que se montaron los engranes fueron de aluminio de 1 pulgada de diámetro. Para futuras aplicaciones, ambos ejes se apoyaron en chumaceras que pueden ser desplazadas para variar la distancia entre centros. En el eje de salida se colocó un freno para ejercer un torque de frenado. Todo el sistema se encuentra montado en una estructura de figura angular de lados iguales. El banco de pruebas ensamblado se presenta en la Figura 1.

Para la medición de esfuerzos se utilizó la película indicadora de presión Fujifilm Prescale ${ }^{\varpi}$. Esta película está formada por hojas con un espesor de 4 a 8 milésimas sensibles al contacto. Cuentan con microcápsulas que al recibir presión dejan una huella de color rosa, que es proporcional a la cantidad de presión que fue requerida para reventar las microcápsulas internas. Cada tipo de película corresponde a un rango de presión, el cual se presenta en la Tabla 1. 


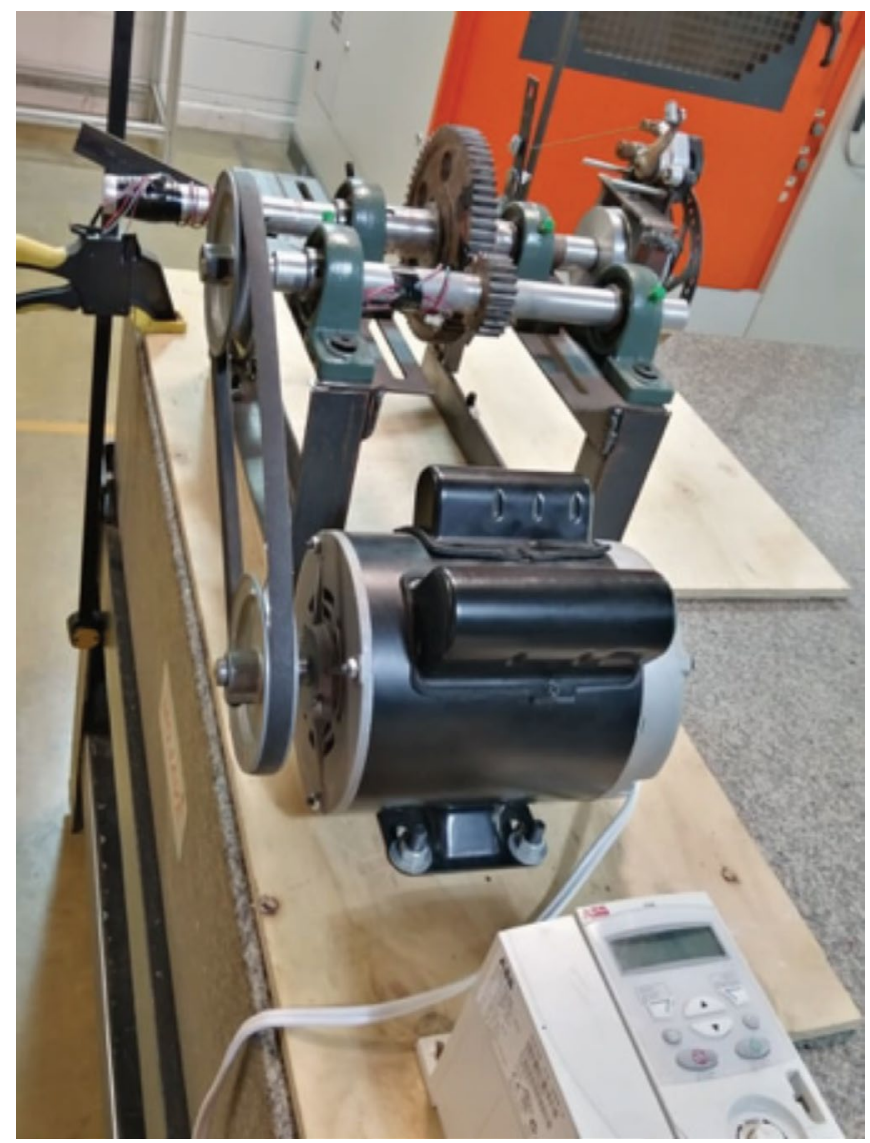

Figura 1. Banco de pruebas.

TABLA 1.

Tipos de Película y Rangos de Presión

\begin{tabular}{|l|l|}
\hline \multicolumn{1}{|c|}{ TIPO DE PELÍCULA } & RANGO DE PRESIÓN (MPa) \\
\hline LLLLW & $0.049-0.193$ \\
\hline LLLW & $0.193-0.588$ \\
\hline LLW & $0.490-2.452$ \\
\hline LW & $2.452-9.81$ \\
\hline MS & $9.81-49.05$ \\
\hline HS & $49.05-127.5$ \\
\hline HHS & $127.5-290.43$ \\
\hline
\end{tabular}

Una vez obtenida la muestra, la película es visualizada y analizada en un escáner con el software FPD8010 WIN, mediante lo cual se pueden ver los resultados en una escala de colores dentro del rango de esfuerzos correspondiente, así como también es posible obtener gráficas trazando perfiles en la zona de interés.

El uso de esta técnica de medición ayuda a obtener una mejor apreciación de la distribución de los esfuerzos de contacto, así como a conocer el valor directo de esfuer- zos sin necesidad de cálculos adicionales, como puede ser el caso de las galgas extensiométricas.

En este trabajo, mediante prueba y error, se determinó que el rango de esfuerzos en los engranes correspondió al tipo de hoja HS y HHS. Las hojas se colocaron en los dientes del engrane rueda. En la Figura 2 se presenta un ejemplo de la película de presión montada en el engrane rueda.

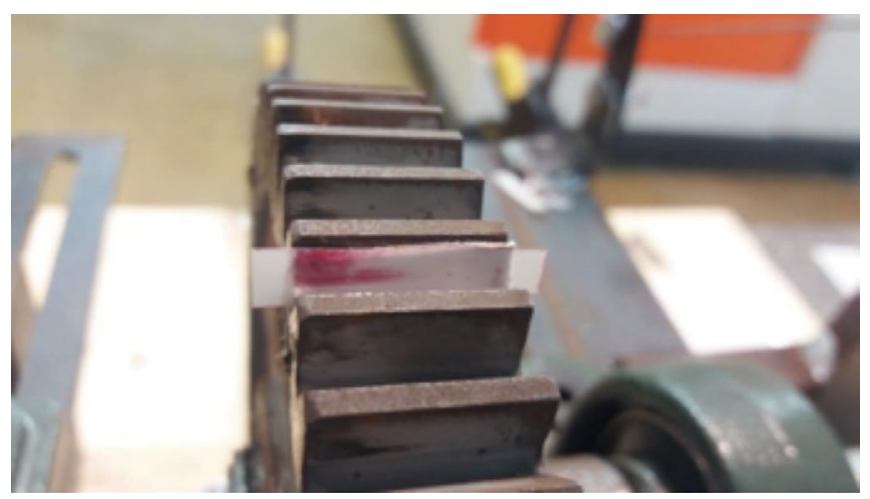

Figura 2. Colocación de película de presión.

Las vibraciones se midieron a través de un acelerómetro triaxial inalámbrico modelo G-Link-200 de la marca Microstrain ${ }^{\oplus}$, que funciona con rangos de entrada de 2-8 G y un ancho de banda de $1 \mathrm{kHz}$ con un alcance de medición de $1 \mathrm{~km}$.

El acelerómetro fue montado en la chumacera del eje de salida del engrane rueda para poder capturar las vibraciones durante el contacto de los engranes, tal como se aprecia en la Figura 3.

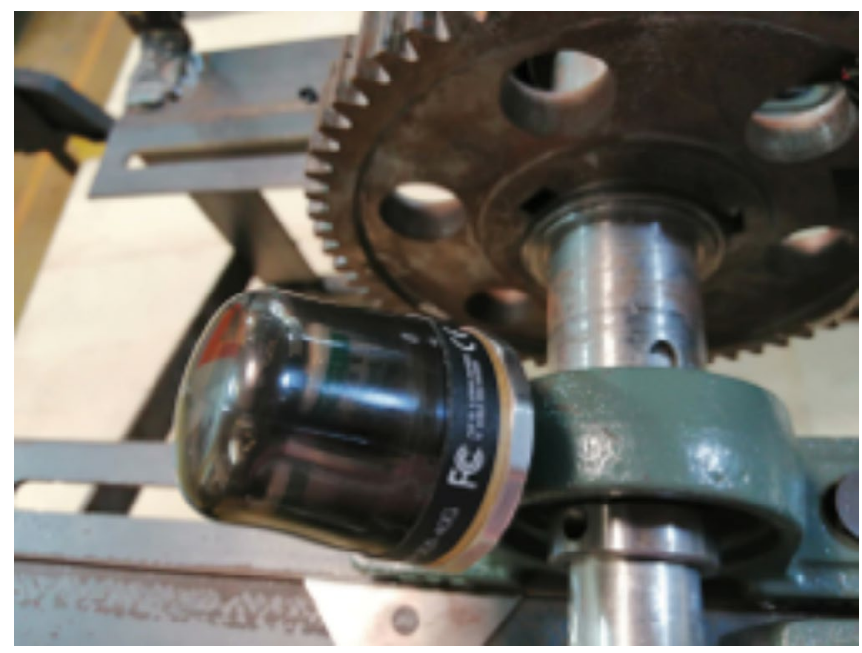

Figura 3. Ubicación del acelerómetro inalámbrico. 
La señal se envió a un receptor WSDA ${ }^{\oplus}$-200-USB conectado a un equipo de cómputo portátil en el que se procesaron los datos recibidos. La aceleración fue medida en $G$ y de salida se obtuvo la amplitud. La señal recibida se encontró en el dominio del tiempo y fue transformada al dominio de la frecuencia aplicando la transformada rápida de Fourier a la señal discreta (Ecuación 1).

$$
A=\sum_{n=0}^{N-1} e^{-i \frac{2 \pi}{N} k n} a_{n}
$$

Todo lo anterior se realizó con el software SensorConnect de uso libre, proporcionado por la empresa fabricante del acelerómetro. Una muestra de la señal recibida en el dominio del tiempo se presenta en la Figura 4.

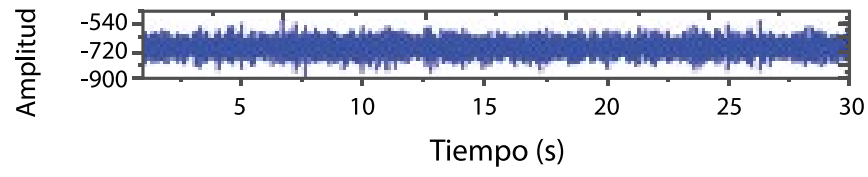

Figura 4. Amplitud en el dominio del tiempo.

Para medir el torque de salida, se utilizaron dos galgas extensiométricas de medio puente colocadas de manera encontrada entre ellas (Figura 5). Las galgas miden la deformación en el eje de salida para después, mediante la Ecuación (2), obtener el torque:

$$
T=\frac{\mu \pi D^{4} E}{192 D(1+v)}
$$

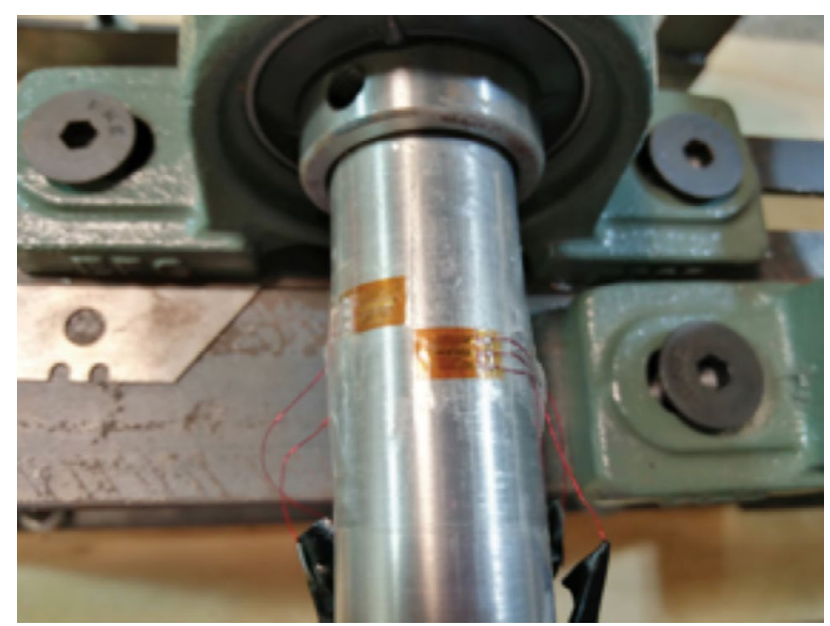

Figura 5. Colocación de galgas para la medición del torque.

Las terminales de las galgas se conectaron a un módulo inalámbrico SG-LINK-200-OEM, en combinación con el receptor WSDA ${ }^{\oplus}$-200-USB y el software SensorConnect.

Se obtuvo una deformación promedio $\mu \varepsilon=-1929.90$ (Figura 6) y aplicando la Ecuación (1) se calculó un torque de $27 \mathrm{Nm}$.

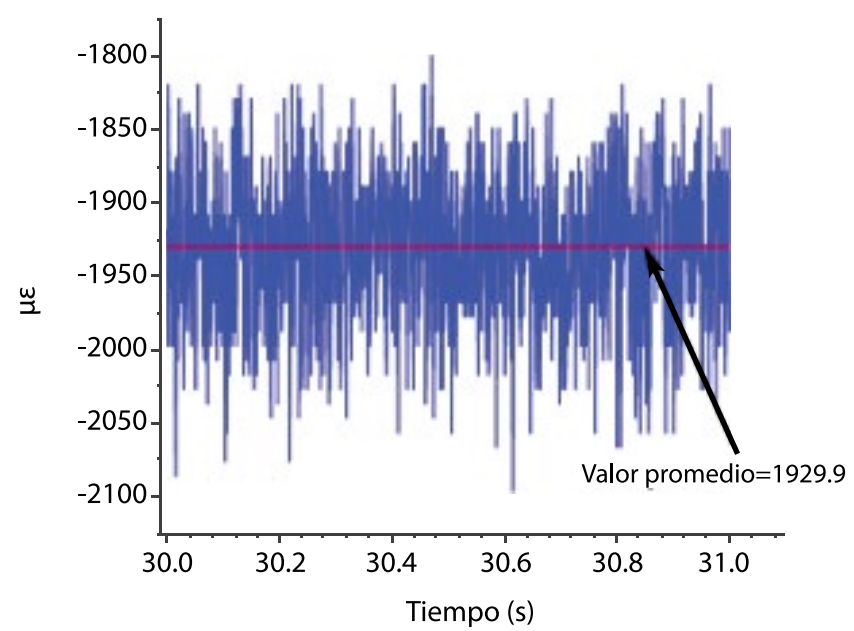

Figura 6. Valor promedio de deformación para el cálculo del torque.

\section{RESULTADOS Y DISCUSIÓN}

Se presentan a continuación los resultados obtenidos de la medición de esfuerzos de contacto y vibraciones en el banco de pruebas de engranes.

Los esfuerzos de contacto en el diente del engrane rueda, sin la aplicación de torque, se presentan en la Figura 7. Las mediciones se realizaron con película de presión en el rango de 50 a $130 \mathrm{MPa}$ (HS) a diferentes velocidades de giro. Se observa una mayor concentración de esfuerzos en el centro del diente cuya magnitud incrementa a medida que aumentan las revoluciones. De acuerdo con la escala, las zonas en color amarillo indican valores mayores al rango superior medido por la película de presión, mientras que los datos en verde indican un valor menor al rango inferior.

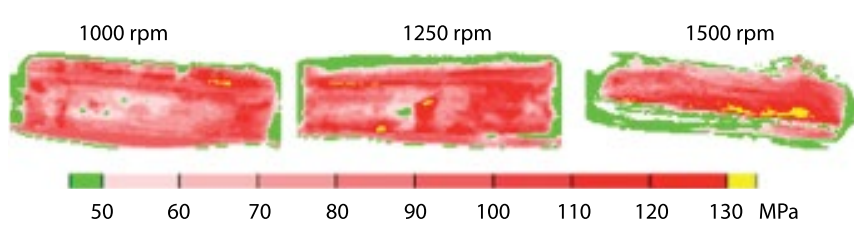

Figura 7. Esfuerzos de contacto en diente del engrane rueda sin carga. 
En la Figura 8 se presentan los esfuerzos de contacto en el diente del engrane rueda con aplicación de torque de frenado. Ahí se observa un incremento considerable en la magnitud de los esfuerzos, mostrando valores superiores a los $300 \mathrm{MPa}$ cuando gira a 1250 y $1500 \mathrm{rpm}$. En el caso de 1000 y 1500 rpm, se observa una zona de color blanco, lo que representa valores mucho menores a $130 \mathrm{MPa}$ que se concentran en el centro del diente, mientras que cerca de la punta ocurre la concentración del máximo esfuerzo.

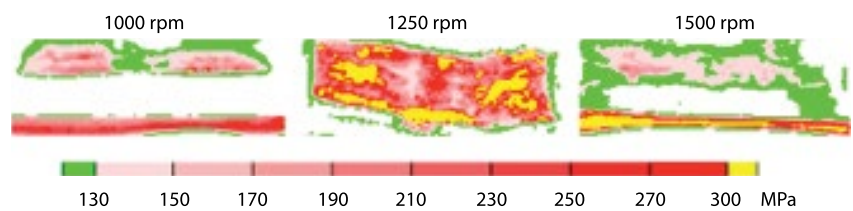

Figura 8. Esfuerzos de contacto en dientes del engrane con carga.

En las Figuras 9 y 10 se muestra la amplitud de vibración de los engranes durante el contacto a diferentes revoluciones, en condiciones sin carga y con carga, respectivamente. La amplitud de los armónicos disminuye en condiciones de carga, sin embargo, la frecuencia es más prolongada durante la aparición de estos. La disminución de la amplitud se debe al efecto del momento de inercia sobre el eje de salida provocado por el torque de frenado, principalmente a altas velocidades, tal como se aprecia en $1500 \mathrm{rpm}$.

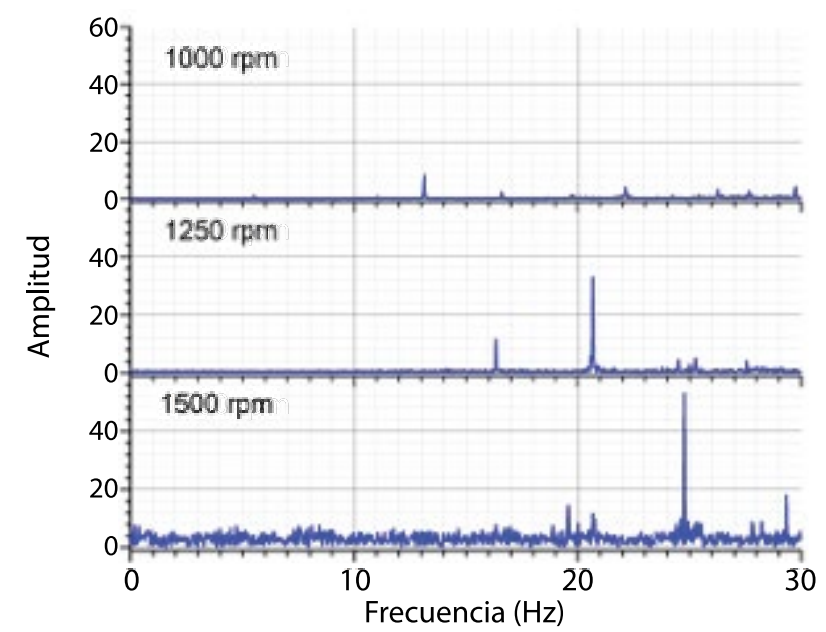

Figura 9. Vibraciones sin carga.

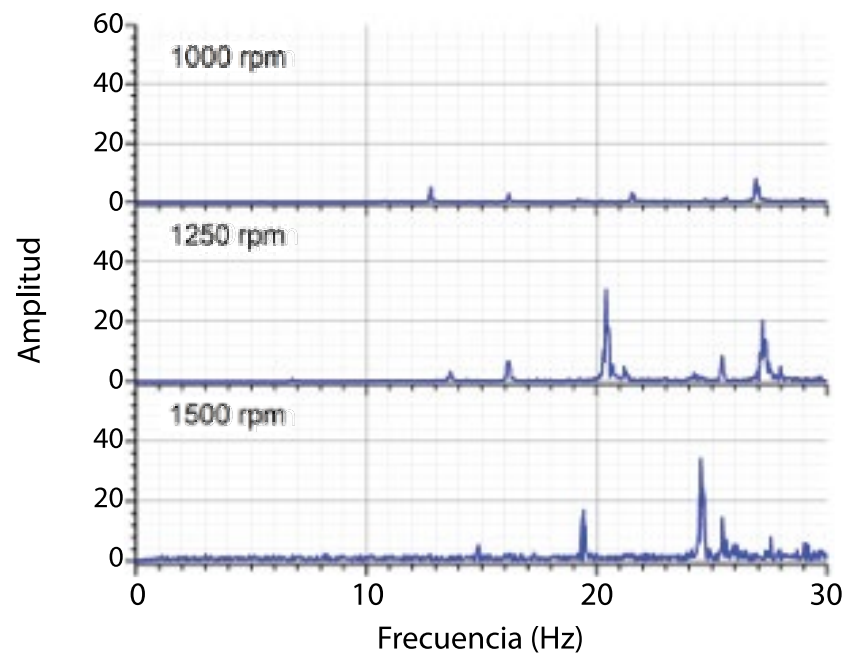

Figura 10. Vibraciones con carga.

\section{CONCLUSIONES}

En este trabajo se presenta la viabilidad del montaje de un banco de pruebas para la medición experimental de esfuerzos de contacto y vibraciones en engranes. Los esfuerzos de contacto obtenidos son consistentes con lo esperado, ya que son mayores al incrementar la velocidad de rotación y al aplicar una carga. Las magnitudes de esfuerzos bajo esas condiciones pueden ser conocidas mediante la aplicación de la técnica de película sensible a la presión. La aplicación del torque de frenado disminuyó la amplitud de las vibraciones, sin embargo, aumentó el tiempo de exposición durante los armónicos.

El uso del banco de pruebas propuesto para el estudio de engranes, combinado con el equipo de sensado propuesto, simplifica y amplía las posibilidades de análisis de esfuerzos y vibraciones en engranes, además de representar una considerable reducción en tiempo y recursos gracias a la simplicidad para la obtención de los datos y a una menor cantidad de componentes empleados.

\section{AGRADECIMIENTO}

Los autores agradecen a la Secretaría de Educación Pública de México, a través del Programa para el Desarrollo Profesional Docente, por el financiamiento brindado a este proyecto a través del convenio 511-6/18-9518. 


\section{REFERENCIAS}

[1] R. G. Budynas y J. K. Nisbett, Shigley's mechanical engineering design, 11. a ed. USA: McGraw-Hill Education, 2019.

[2] G. Mehta, M. Somani, T. N. Babu y T. Watts, “Contact Stress Analysis on Composite Spur Gear using Finite Element Method", materialstoday: proceedings, vol. 5, no. 5, pp. 13585-13592, junio 2018. https://doi.org/10.1016/j.matpr.2018.02.354

[3] Y. Wang, Y. Liu, W. Tang y P. Liu, "Parametric finite element modeling and tooth contact analysis of spur and helical gears including profileand lead modifications", Engineering Computations, vol. 34, no. 8, pp. 28772898, noviembre 2017. https://doi.org/10.1108/EC-062016-0203
${ }^{[4]}$ S. S. Patil, S. Karuppanan y I. Atanasovska. "Experimental measurement of strain and stress state at the contacting helical gear pairs", Measurement, vol. 82, pp. 313322, Marzo 2016. https://doi.org/10.1016/j.measurement.2015.12.046

${ }^{[5]}$ K. G. Raptis y A. A. Savaidis, "Experimental investigation of spur gear strength using photoelasticity", Procedia Structural Integrity, vol. 10, pp. 33-40, octubre 2018. https://doi.org/10.1016/j.prostr.2018.09.006

[6] D. Hartono, D. Halim y G. W. Roberts. "Gear fault diagnosis using the general linear chirplet transform with vibration and acoustic measurements", Journal of Low Frequency Noise, Vibration and Active Control, vol. 38, no. 1, pp. 36-52, marzo 2019. https://doi. org/10.1177/1461348418811717 